\title{
Hasél II o La persecución penal de la inquina (a propósito de un texto de Jacobo Dopico)
}

Hasél II or The criminal prosecution of animosity (about a text by Jacobo Dopico)

\author{
Víctor Javier Vázquez Alonso \\ Universidad Sevilla \\ ORCID ID 0000-0003-3494-3827 \\ vvazquez@us.es
}

\section{Cita recomendada:}

Vazquez Alonso, V. J. (2021). Hasél II o La persecución penal de la inquina (a propósito de un texto de Jacobo Dopico). Eunomía. Revista en Cultura de la Legalidad, 21, pp. 392-398. doi: https://doi.org/10.20318/eunomia.2021.6356

Recibido / received: 15/09/2021

\begin{abstract}
Resumen
Este trabajo analiza brevemente las sombras del sistema de protección de la libertad de expresión en España al hilo del comentario a la sentencia del Tribunal Supremo nº 135/2020, de 7 de mayo, realizado por el profesor Jacobo Dopico en el anterior número de esta revista. Este caso resuelve el recurso interpuesto por el rapero Pablo Hásel. En el trabajo se analiza, en primer lugar, los factores que han llevado al viraje restrictivo en nuestra idea de la libertad de expresión para, en segundo lugar, ilustrar con el caso Hásel las consecuencias de esta deriva.
\end{abstract}

\section{Palabras clave}

Libertad de expresión, Pablo Hásel, discurso de odio.

\begin{abstract}
This paper briefly examines the grey areas of the freedom of speech protection system in Spain in connection with the review of the Spanish Supreme Court ruling (number 135/2020) of 7 May 2020 carried out by Jacobo Dopico in the last issue of this journal. This case decides the appeal of the rap artist Pablo Hásel. First, this paper analyzes the factors that led to a restrictive turn in our idea of freedom of speech in order to, subsequently, show the consequences of this trend in the light of Hásel's case.
\end{abstract}

\section{Keywords}

Freedom of speech, Pablo Hásel, hate speech. 
La dirección de la revista Eunomia me invita a la difícil tarea de dar respuesta a un trabajo del apreciado colega Jacobo Dopico (2021) sobre una de esas resoluciones judiciales que nacen condenadas a la atención mediática y al debate público. Estamos hablando de la sentencia de la Sala II del Tribunal Supremo ${ }^{1}$ que resuelve el recurso interpuesto por el ya célebre rapero Pablo Hasél, frente a una previa condena por parte de la Audiencia Nacional, por la comisión de los delitos de enaltecimiento del terrorismo, injurias a la corona e injurias a la policía. Es determinante, sin duda, para la popularidad de este litigio el hecho de que la comisión del delito de enaltecimiento implicara a la postre la entrada en prisión del condenado, y también, el propio hecho de que el mismo Hasél ya hubiera sido condenado por delitos de expresión con anterioridad, en otro polémico fallo.

Pero, volviendo al comienzo, señalaba que la tarea de dar respuesta al profesor Dopico se antoja difícil y la razón de ello, fundamentalmente, es que suscribo de forma plena las conclusiones del análisis que él lleva a cabo en el número anterior de esta publicación, donde apunta, en un sentido muy crítico, a la ausencia de tipicidad de las expresiones que fueron consideradas constitutivas de los tres delitos señalados por parte del Tribunal. Me es imposible, en este sentido, responder sensu stricto a las tesis esbozadas por el profesor Dopico, como también, por pura deferencia hacia el lector, redundar en ideas que ya han sido formuladas por él de forma precisa y que comparto.

A este respecto, y valiéndonos del caso Hasél II y de la propia glosa llevada a cabo por nuestro colega penalista, intentaré aquí brevemente dar cuenta de algunos de los síntomas que ponen de manifiesto, en mi opinión, la zona de sombra por la que transita nuestro sistema de libertad de expresión. Síntomas, en cualquier caso, que, como insistiré, no dejan de verse bien representados en este litigo.

Desde hace años asistimos a un incremento de las condenas por delitos de expresión en España que es paralelo a una nueva comprensión, más restricta, del ámbito protegido por el artículo 20 de nuestra Constitución. Tomando en consideración la jurisprudencia en casación, y la propia doctrina constitucional, muy especialmente, por lo reciente y significativa, la STC $190 / 2020^{2}$, creo que podemos afirmar que se corre el riesgo de abdicar, ya sea parcialmente, en algunos de los presupuestos epistemológicos que sostenían, en nuestro ordenamiento, lo que podríamos denominar un concepto fuerte, en sentido democrático, de libertad de expresión. Desde mi punto de vista, a esta situación se llega por la confluencia de varios factores que dejan su impronta en la propia sentencia Hasél II.

El primero de ellos, por lo menos en el tiempo y creo que en relevancia, es el de la tipificación en el ordenamiento español del delito de enaltecimiento del terrorismo. Esta decisión de política criminal ha tenido, en mi opinión de forma inequívoca, implicaciones generales en la propia comprensión jurídica de la peligrosidad abstracta del discurso público, desvinculando el juicio sobre el carácter lesivo de ciertas manifestaciones de elementos empíricos y constatables desde una lógica causal. Con la tipificación del delito de enaltecimiento del terrorismo ${ }^{3}$, y a pesar de la explícita acotación del tipo que, también a la luz del derecho europeo ${ }^{4}$, ha hecho

\footnotetext{
${ }^{1}$ STS no 135/2020, de 7 de mayo.

2 Para un análisis «en caliente» de la fragilidad argumental de esta sentencia, véase, el comentario de Valero Heredia, A. (17 de enero de 2021).

${ }^{3}$ Ley Orgánica 7/2000, de 22 de diciembre, de modificación de la Ley Orgánica 10/1995, de 23 de noviembre, del Código Penal.

${ }^{4}$ En especial, la Directiva UE 2017/541 sobre lucha contra el terrorismo que, entre otras cosas, señala que «Esta conducta debe tipificarse cuando conlleve el riesgo de que puedan cometerse actos terroristas. En cada caso concreto, al examinar si se ha materializado ese riesgo se deben tener en cuenta las circunstancias específicas del caso, como el autor y el destinatario del mensaje, así como el
} 
la jurisdicción constitucional, lo cierto es que se abrió ${ }^{5}$ la puerta a un análisis conjetural sobre la incidencia abstracta que determinadas expresiones pueden tener en la creación o consolidación de un ambiente propicio para la comisión de ilícitos. A una cultura que, podríamos decir, estima de forma generosa la competencia del derecho penal frente al discurso socialmente nocivo. En este sentido, como se insistirá, el litigio que aquí se examina da buena cuenta de cómo dicha cultura prevalece, en ocasiones, sobre la propia restauración que el juez constitucional ha llevado a cabo del elemento incitador en la aplicación del tipo penal de enaltecimiento.

Al impacto que tuvo en la comprensión de la libertad de expresión la tipificación del enaltecimiento se suma aquella que produce la irrupción en nuestro acervo jurídico del concepto «discurso del odio» ${ }^{6}$. A este respecto, creo que bien puede decirse que, lejos de encontrarnos ante un concepto jurídico indeterminado, nos hallamos más bien ante una suerte de «concepto jurídico de efectos indeterminados», y ello es así porque, más allá del hecho de que se haya incorporado a nuestro ordenamiento un tipo penal específico como el del artículo $510 \mathrm{CP}$, la idea de discurso odioso ha sido un argumento recurrente a la hora de excluir del ámbito protegido por la libertad de expresión ciertas manifestaciones, instaurándose, de este modo, una suerte de «test de la inquina», en cuya aplicación, muy a menudo, lejos de contrastarse la adecuación real de ciertas expresiones para poner en riesgo determinados bienes jurídicos, o siquiera para generar una atmósfera favorable a la discriminación de colectivos históricamente preteridos y con dificultades materiales para hacer valer sus derechos, se ha juzgado, en último término, lo que podríamos calificar como la mismidad del odio, es decir, la presencia de un sentimiento de desprecio en el emisor del discurso?. El binomio prohibición del discurso del odio y tutela de las minorías ha sido sustituido en muchas ocasiones por una argumentación donde la constatación de la inquina tras una determinada expresión es considerada presupuesto para situar extramuros de la libertad de expresión ciertas manifestaciones y, simultáneamente, y casi como consecuencia obligada, considerar su tipicidad.

Cabría valorar, junto a las razones expuestas, y también con relación al contexto en el que se produce la mutación en nuestra comprensión de los límites o, si se prefiere, del propio sistema de libertad de expresión, la circunstancia de que, tanto en la jurisprudencia constitucional, como en la propia doctrina judicial del Tribunal Europeo de Derechos Humanos, convive una suerte de miscelánea de teoría de los derechos fundamentales (Caruso, 2017), alternando ambos tribunales patrones de argumentación basados en la ponderación y, por lo tanto, en una comprensión principialista del derecho a la libertad de expresión, con otros en los que los límites a esta libertad se dirimen desde una perspectiva interna, es decir, delimitando de forma más o menos estricta su ámbito de protección y situando, por lo que ahora nos atañe, ciertos discursos extremos fuera del ámbito protegido. Desde luego, no son estas páginas el lugar adecuado para terciar en la que es ya una de las polémicas patrias de más rico abolengo doctrinal, la que enfrenta, podríamos decir en broma, a

contexto en el que se haya cometido el acto. También deben considerarse la importancia y la verosimilitud del riesgo al aplicar la disposición sobre provocación pública de acuerdo con el Derecho nacional»

${ }^{5}$ Así, la conocida STC 112/2016, integrando en la aplicación del tipo la constatación del riesgo provocado por las expresiones enaltecedoras y su idoneidad para la incitación.

${ }^{6}$ Una interesante lectura favorable a la introducción de este tipo, con especial atención a su trasfondo ético, puede verse en Pablo Serrano, (2017).

${ }^{7}$ Qué mejor ejemplo, en este sentido, que la STC 177/2015, de 22 de julio, donde, como es sabido, es el concepto de «discurso del odio», el que da estructura a la argumentación utilizada por el Tribunal Constitucional para avalar la condena por injurias a la Corona que la Audiencia Nacional impuso con motivo de la quema de fotos del Rey. 
alicantinos y asturleoneses ${ }^{8}$ (Atienza, 2020), pero creo que sí puede constarte que esa impureza teórica es presupuesto para una cierta imprevisibilidad judicial ${ }^{9} \mathrm{y}$, concretamente, como hemos visto, para que el uso de la idea de discurso odioso sirva, a la postre, no sólo como criterio de exclusión del ámbito protegido por el derecho a la libertad de expresión, sino también como argumento a favor de la tipicidad $^{10}$.

De otro lado, cabría señalar que la funcionalización de la libertad de expresión, en tanto derecho comprendido al servicio de la formación de la opinión pública, es decir, desde su valor político o democrático, ha servido en ocasiones como presupuesto para su interpretación limitativa, al restringirse dicha dimensión democrática a aquellos discursos que respondan a un patrón de decoro, sentándose así la premisa, muy cuestionable, de que el exabrupto, el leguaje extremo o desbarrado, quedan al margen de la tutela del derecho fundamental (Urias, 2021). Así, a falta de una teoría de la libertad de expresión que tome en cuenta la dimensión puramente subjetiva de ésta, es decir, su vinculación a la autorrealización del individuo ${ }^{11}$, la delimitación estricta de aquello que puede considerarse bajo el marchamo del discurso público condiciona progresivamente, en sentido restrictivo, el alcance de lo protegido por el derecho a la libertad de expresión.

A lo anterior hay que añadir que se ha producido igualmente, en este ámbito, una cierta banalización, en buena medida inédita, de la vinculación que nuestro ordenamiento prescribe a la doctrina judicial del Tribunal Europeo de Derechos Humanos. A este respecto, además de lecturas parciales o incoherentes con respecto a dicha jurisprudencia, tenemos ejemplos recientes y significativos de desacatos hacia la misma. En este sentido, la reformulación restrictiva de nuestra comprensión de los límites de la libertad de expresión implica hoy una relación de tensión, sobre todo en lo relativo a la tutela penal específica de instituciones y símbolos ${ }^{12}$, con la que es una doctrina consolidada en la jurisprudencia de Estrasburgo ${ }^{13}$.

En cualquier caso, estos presupuestos jurídicos del viraje restrictivo en nuestra idea de la libertad de expresión no se sostendrían al margen de un cambio cultural de fondo. Cambio que, creo, tiene que ver, en primer lugar, con una nueva comprensión de los riesgos inherentes a ciertos discursos en un contexto tecnológico que hace posible, de forma hasta ahora inédita, la viralidad. Más allá del debate, siempre inconcluso, sobre la resistencia del marco conceptual de nuestro sistema de libertad de expresión e información a la disrupción tecnológica que supone la comunicación a través de la red (Boix, 2017), creo que cabe constatar que, por lo menos en el ordenamiento español, se ha producido un acto reflejo de caución judicial que ha afectado, entre otras cosas, a la comprensión de los límites al humor, a la sátira

\footnotetext{
${ }^{8}$ En concreto, una interesante crítica a la lógica ponderativa aplicada en el ámbito de la libertad de expresión, a propósito de la STC 35/2020, la podemos leer en García Amado, A. (2 de abril de 2020). Entre los autores que se caracterizan por abogar por una teoría interna de los límites a la libertad expresión, puede verse, Urías (2019) o Presno Linera (2017).

${ }^{9}$ Si hay un trabajo que aborda esta cuestión, desde la perspectiva de la teoría de los derechos, y reivindicando, frente a los autores arriba citados, el necesario análisis de proporcionalidad y, por lo tanto, la no exclusión a priori de la cobertura constitucional de ciertos discursos, ese es el de Alcácer Guirao (2018).

${ }^{10}$ Alcácer Guirao lo ha resumido muy bien: «Si la primera consecuencia rechazable de la jurisprudencia constitucional es la omisión del juicio de proporcionalidad, la segunda es la justificación automática y acrítica de la sanción penal» Alcácer Guirao (2018). En extenso, puede verse su reciente trabajo, La libertad del odio, Discurso intolerante y protección penal de minorías, Alcácer Guirao (2020).

${ }^{11}$ Algo que propone, entiendo, Teruel Lozano (2018).

12 Otegi Mondragón c. España, de 15 de marzo de 2011; Stern Taulats y Roura Capellera c. España, de 13 de marzo de 2018.

${ }^{13}$ Recomiendo la lectura del reciente estudio de Álvarez Rodríguez, I. (2020). Igualmente, puede verse, Presno Linera (2020).
} 
religiosa o al derecho de protesta... De otro lado, entiendo que también incide en este retroceso la progresiva consolidación de una conciencia social en torno al pretendido «derecho» a no sentirse ofendido, es decir, la extensión de la creencia de que el ordenamiento nos ofrece instrumentos para poner fin a aquellos discursos que hieren nuestros sentimientos. Llama la atención también, y con razón, el profesor Jacobo Dopico, sobre la «hoolliganizacion» del debate público en torno a aquellos litigios relacionados con los límites de la libertad de expresión. Elemento este último que, del mismo modo, contribuye a que la «ecología» de la libertad de expresión sea más propicia para una lectura restrictiva de sus contornos.

El caso Hasél II, como señalábamos en un principio, se inscribe dentro de este contexto jurídico y cultural, siendo a su vez un ejemplo excepcional de las consecuencias prácticas del mismo. Ahora bien, al contrario de lo comúnmente asumido en la discusión informal que, sobre el litigio, se ha producido en los medios, no se trataba aquí del enjuiciamiento de un artista en tanto tal, es decir, a través de su obra, sin que, por lo tanto, sea preciso aproximarse a las expresiones litigiosas con el filtro de análisis necesario de la libertad artística, el cual, como he podido defender en alguna ocasión (Vázquez 2017), exige tomar en consideración la especificidad de este código y relativizar, en mi opinión, la lesividad de aquellas expresiones distorsionadoras o extremas que hayan podido usarse en una creación. La idea del juicio al rapero confunde así, en mi opinión, lo que en puridad se sometía a escrutinio, que no es sino la relevancia penal de una reiterada, extrema e incontinente crítica a través de la red, en concreto a través de tuits, llevada a cabo por un sujeto de relativa celebridad en determinados ámbitos contestatarios.

A este respecto, e intentando cumplir con la palabra dada de no ser reiterativo o redundante con el comentario a la sentencia del profesor Dopico, con el cual ya he señalado que coincido plenamente, creo que la argumentación esgrimida en casación para confirmar la condena impuesta a Pablo Hasél no consigue explicar con claridad tres puntos esenciales.

En primer lugar, y respecto al delito de injurias a la Corona, en la sentencia no se explica con suficiencia, por qué no es en este caso atendible la vinculante doctrina del Tribunal Europeo de Derechos humanos que, reiteradamente, y con España como excusa, ha advertido de la difícil compatibilidad con el Convenio Europeo de Derechos Humanos de tipos penales que impidan en una sociedad democrática ejercer con amplitud el derecho de crítica a las instituciones estatales, especialmente a la jefatura del Estado, sea cual sea la forma política de este. No consigo ver, a este respecto, en el compendio de arrebatos antimonárquicos que son objeto de juicio, y de los cuales trasciende un juicio de valor radicalmente despreciativo hacia la monarquía ${ }^{14}$, un comportamiento que pueda calificarse de típico, considerando tanto el amparo que la libertad de expresión nos ofrece para hacer público nuestro juicio más extremo de una institución, como los propios límites con los que el derecho penal ha de proyectarse sobre esta libertad pública.

En segundo lugar, y con relación al enaltecimiento, la apreciación de la tipicidad de ciertas expresiones se lleva a cabo con el peaje de obviar o de no interpretar en su justa medida, el alcance de una jurisprudencia constitucional ya

\footnotetext{
${ }^{14}$ Así, entre otros asertos, se toman en consideración algunos como: «El mafioso del Borbón de fiesta con la monarquía saudí, entre quienes financian el ISIS queda todo», «La Monarquía mafiosa que da lecciones a países donde nadie es desahuciado»; «Los amigos del reino español bombardeando hospitales mientras Juan Carlos se va de putas con ellos»; " Mientras llaman terrible tiranía a Cuba donde con menos recursos no se desahucia, ocultan los negocios mafiosos del Borbón en Arabia Saudí» o «El estado español dando armas a los criminales amigos de la monarquía para que puedan bombardear Yemen. Que se sepa».
} 
reiterada que vincula la aplicación del tipo penal del enaltecimiento a un análisis causal en el que se coteje de forma estricta, y no de manera conjetural ${ }^{15}$, la idoneidad de las expresiones para «propiciar o alentar, aunque sea de manera indirecta, una situación de riesgo para las personas o derechos de terceros o para el propio sistema de libertades», quedando, por lo tanto, fuera del ámbito del reproche penal «la mera adhesión ideológica a posiciones políticas de cualquier tipo, que resultaría plenamente amparada por el art. 16 CE y, en conexión, por el art. 20 CE».

Finalmente, y con relación a la condena sobre la base del Art. 504.2 CP, es significativa, en mi opinión, y tomando en consideración el contenido de las expresiones litigiosas, la taxatividad con la que el Tribunal descarta que dichas expresiones no se encuentren bajo el paraguas de la libertad de expresión, en este caso manifestada como una virulenta crítica policial, que, por mucho que nos pueda resultar desmedida o injusta, no aparece en el caso concreto desconectada de sucesos o procesos penales de inequívoca relevancia pública, que en su momento suscitaron controversia, y en los que es la actividad de los cuerpos y fuerzas de seguridad del Estado el elemento objetivo de la discusión. A este respecto, tampoco está de más recordar que el propio Tribunal Europeo de Derechos Humanos ha insistido en el amparo expreso que ofrece el Convenio al discurso antipolicial ${ }^{16}$ ya que, al fin y al cabo, nos encontramos ante una crítica al poder público, siendo, en este sentido, necesario comprender o delimitar de forma minimalista la "dignidad" institucional de estos cuerpos de seguridad que es, en último término, el valor que el tipo penal protege.

Como cierre a esta respuesta, que no es tal, al profesor Dopico, me gustaría destacar una conclusión que se desprende bien del comentario que él lleva a cabo de la sentencia, y es que la devaluación en la comprensión constitucional de la libertad de expresión, la quiebra de algunos de sus presupuestos epistemológicos ha provocado a su vez una redimensión del derecho penal en este ámbito. El caso Hasél II demuestra, en este sentido, que el cambio que se produce en nuestra cultura constitucional a la hora de entender qué es y qué no discurso protegido, es paralelo también a una relativización de ciertos principios basilares del derecho penal, dentro de este ámbito de los delitos de expresión, que, en último término, tiene como consecuencia que nuestros jueces operen con juicios subsuntivos demasiado favorables a la hora de considerar la tipicidad penal de ciertas expresiones. Todo ello con el peaje no menor de colmar de celebridad a sus autores.

\section{Bibliografía}

Alcácer Guirao, R. (2018). Opiniones Constitucionales. Indret: Revista para el Análisis del Derecho, 1, pp. 1-39.

Alcácer Guirao R. (2020). La libertad del odio, Discurso intolerante y protección penal de minorías. Madrid: Marcial Pons.

Álvarez Rodríguez, I. (2020). De la libertad de expresión en España: Una reflexión al hilo de la jurisprudencia del TEDH. Anales de Derecho, Número especial AdD: El TEDH en su sesenta aniversario.

Atienza Rodríguez M., García Amado A. (2020). Um debate sobre a ponderaçao. Barcelona: J.M. Bosch Editor.

Boix Palop A. (2016). La construcción de los límites a la libertad de expresión en las redes sociales. Revista de estudios políticos, 173, pp. 55-112.

\footnotetext{
${ }^{15}$ Véase, a este respecto, el muy reciente trabajo de Valero Heredia, A. (2021).

${ }^{16}$ Savva Terentyev c. Rusia. Sentencia de 28 de agosto de 2018.
} 
Caruso, C. (2017). El hate speech en Estrasburgo. El pluralismo militante de sistema convencional. En L. Alonso y V. J. Vázquez. (eds.), Sobre la libertad de expresión y el discurso del odio. Textos críticos (pp. 103-121). Sevilla: Athenaica.

Dopico Gómez-Aller, J. (2021). El segundo "caso Pablo Hasél". Eunomía. Revista en Cultura de la Legalidad, 20, pp. 393-414.

García Amado, A. (2 de abril, 2020). O de la eterna cuestión de si el TC es un tribunal de supercasación. Almacén de Derecho. Recuperado de https://almacendederecho.org/sobre-la-sentencia-del-tc-352020-en-el-conocidocomo-caso-strawberry

Pablo Serrano, A. (2017). Límites jurídico-penales del discurso (puro) del odio. En L. Alonso Sanz, V. J. Vázquez Alonso (Eds.), Sobre la libertad de expresión y el discurso del odio. Textos críticos (pp. 145-160). Sevilla: Athenaica.

Presno Linera, M. y Teruel Lozano, G. (2017). La libertad de expresión en América y Europa. Lisboa: Juruá Editora.

Presno Linera, M. (2020). La libertad de expresión según el Tribunal Europeo de Derechos Humanos. Revista de la Facultad de Derecho de México, 70, 276, pp. 461-492.

Teruel Lozano G. (2018). Cuando las palabras generan odio: límites a la libertad de expresión en el ordenamiento constitucional español. Revista Española de Derecho Constitucional, 38, 114, pp. 13-45.

Urías, J. (2019). Libertad de expresión: Una inmersión rápida. Barcelona: Tibidabo.

Urías, J. (2021). Insultos en el Tribunal Constitucional (sobre un requisito para el ejercicio de las libertades de expresión e información). Revista Española de Derecho Constitucional, 121, pp. 271-301.

Valero Heredia, A. (2021). La Sentencia del Tribunal Constitucional 35/2020, en el caso Strawberry, un paso más, aunque no el definitivo, hacia la desaparición del delito de enaltecimiento del terrorismo. Revista Española de Derecho Constitucional, 122, pp. 367-388

Valero Heredia, A. (17 de enero de 2021). A puta bandeira o la autodestrucción de un Tribunal. AlRevésyAlDerecho. Recuperado de http://blogs.infolibre.es/alrevesyalderecho/?p=5957

Vázquez Alonso, V. J. (2017). Artistas abyectos y discurso del odio. En L. Alonso y V. J. Vázquez. (eds.), Sobre la libertad de expresión y el discurso del odio. Textos críticos (pp. 211-231). Sevilla: Athenaica. 